\title{
El caso de 'viste': ¿Un nuevo marcador discursivo rioplatense?
}

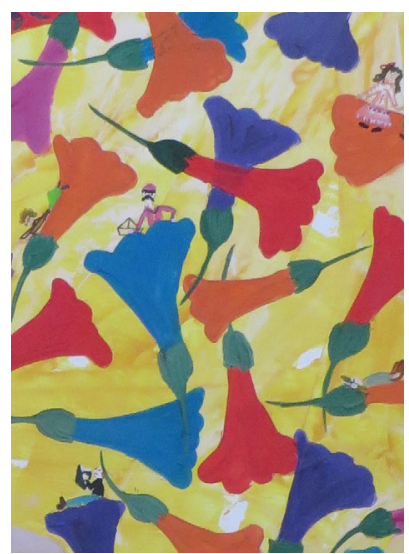

Irina Lifszyc

Universidad de Massachusetts Amherst, Estados Unidos

ilifszyc@umass.edu

Trabajo recibido el 4 de abril de 2021 y aprobado el 13 de julio de 2021.

\section{Resumen}

El estudio del proceso de creación de los marcadores discursivos en distintas lenguas ha proliferado recientemente. Si bien los diversos autores enmarcan este cambio lingüístico dentro de distintas teorías, lo cierto es que estos trabajos se han multiplicado especialmente en español, una lengua cuyos verbos presentan tal flexibilidad que permite su recategorización como partículas del discurso. El objetivo de este trabajo es estudiar la evolución diacrónica del significado de viste en español rioplatense y su uso como marcador discursivo. Se extrajeron datos de tres corpus electrónicos de la Real Academia Española y, tras descartar los ejemplos de viste que correspondían al presente del verbo vestir, se analizaron todas las instancias de viste según se utilizaran como verbo principal o marcador discursivo. Además, en los casos en que viste se utilizó como verbo principal, se evaluó si su significado era el etimológico (la percepción a través de los ojos) o epistémico (conocimiento), expansión semántica que habría permitido su uso como marcador discursivo. Se encontró que, a lo largo del siglo $\mathrm{XX}$, viste experimentó un proceso de debilitamiento semántico que le añadió un significado epistémico, lo que luego permitió que los hablantes manipularan la forma pragmáticamente y la utilizaran como marcador discursivo.

Palabras clave: marcador discursivo, viste, gramaticalización, debilitamiento semántico, español rioplatense 


\title{
The case of 'viste': A new discourse marker in Rioplatense Spanish?
}

\begin{abstract}
The study of the development of discourse markers has recently bloomed, particularly in Spanish, whose verbs are highly flexible and can be easily recategorized as discourse particles. The goal of this paper is to analyze the diachronic evolution of the meaning of viste (preterit of Spanish verb ver 'to see') in Rioplatense Spanish and its use as a discourse marker. Tokens of viste were collected from three electronic corpora developed by the Real Academia Española (the Royal Spanish Academy). After discarding those tokens of viste whose meaning was the present tense of verb vestir 'to wear', tokens of viste corresponding to the preterit of verb ver 'to see' were analyzed by function: main verb or discourse marker. Also, when viste was acting as the main verb of the sentence, its meaning was studied to assess if it was etymological (perceiving something through the eyes) or epistemic (knowledge, awareness). Such semantic bleaching could have allowed speakers to use viste as a discourse marker. Results show that throughout the 20th century viste has indeed experienced semantic bleaching which added another layer of meaning to the etymological meaning. Such an added layer of meaning allowed speakers to and use viste as a discourse marker.
\end{abstract}

Keywords: discourse marker, viste, Rioplatense Spanish, semantic bleaching

\section{O caso de 'viste': Um novo marcador discursivo do Rio da Prata?}

\section{Resumo}

O estudo do processo de criação de marcadores discursivos em diferentes línguas se proliferou recentemente. Embora diferentes autores enquadram essa mudança linguística em diferentes teorias, a verdade é que esses estudos se multiplicaram, principalmente em espanhol, uma língua cujos verbos são tão flexíveis que podem ser recategorizados como partículas do discurso. O objetivo deste trabalho é analisar a evolução diacrônica do significado de viste no espanhol do Rio da Prata e seu uso como marcador discursivo. Os dados foram extraídos de três corpora eletrônicos da Real Academia Espanhola e, após o descarte dos exemplos de viste que correspondiam ao tempo verbal presente do indicativo do verbo vestir, todas as instâncias de viste foram analisadas conforme sua utilização como verbo principal ou marcador discursivo. Além disso, nos casos em que viste era usado como verbo principal, avaliou-se se seu significado era etimológico (percepção através dos olhos) ou epistemológico (conhecimento), expansão semântica que teria permitido seu uso como marcador discursivo. Ao longo do século 20, descobriu-se que viste passou por um processo de enfraquecimento semântico que lhe adicionou um significado epistemológico, o que permitiu aos falantes manipular a forma pragmaticamente e usá-la como um marcador discursivo.

Palavras-chave: marcador discursivo, viste, gramaticalização, enfraquecimento semântico, espanhol do Rio da Prata 


\section{Introducción}

Los marcadores discursivos son expresiones independientes de la estructura sintáctica de la oración que señalan la relación entre los distintos segmentos del discurso para darle cohesión al texto y guiar al oyente hacia la correcta interpretación del mensaje según las intenciones del emisor (Garcés Gómez 2008; Fraser 2009; Portolés Lázaro 2015). Estas partículas se originan a partir de diferentes clases de palabras - verbos, adjetivos, adverbios-que han experimentado diversos procesos a lo largo del tiempo mediante los cuales adquirieron independencia sintáctica y significados más subjetivos, lo que permitió que funcionaran fuera de la estructura sintáctica oracional y se constituyeran como partículas pragmáticas (Company Company 2004; Garcés Gómez 2008).

En los últimos años, ha aumentado la cantidad de trabajos que se enfocan en el estudio del proceso de creación de marcadores discursivos en muchas familias de lenguas, desde lenguas germánicas (Lindström y Wide 2005; Van Olmen 2012; Brinton 2017; Meng 2019) hasta lenguas romances (Waltereit 2002; Vincent 2005; Fagard 2012). Si bien los académicos que han estudiado este cambio lingüístico lo enmarcan dentro de distintas teorías, lo cierto es que estos trabajos se han multiplicado especialmente en español (Ocampo 2006; Ocampo 2009; Mondaca Becerra, Méndez Carrasco y Rivadeneira Valenzuela 2015; Tanghe 2016; Lazo García 2016; Azofra Sierra y Enghels 2017). Los verbos de esta lengua, según señala Company Company (2004), presentan tal flexibilidad que pueden recategorizarse como partículas del discurso; es decir, las diferentes formas verbales que los hablantes utilizan en determinados contextos se recargan de subjetividad, lo que debilita su significado etimológico y permite expandir el contexto de aplicación de dichas formas. Finalmente, estas formas adquieren mayor independencia sintáctica y, en muchas ocasiones, resultan en un marcador discursivo (Company Company 2004).

El objetivo de este trabajo es analizar la evolución diacrónica del significado de viste en español rioplatense y su uso como marcador discursivo. Resulta particularmente interesante estudiar el uso de marcadores discursivos de una comunidad dialectal como el español del Río de la Plata, comunidad en la que el voseo pronominal y verbal constituye la norma culta y la variedad de prestigio del país (Fontanella de Weinberg 1999, citado en García Negroni y Ramírez Gelbes 2010). Esto se ve en el uso de marcadores cuyo análisis diacrónico fue realizado por otros autores, como mirá (Ocampo 2009), un marcador proveniente del imperativo del verbo mirar en la segunda persona del singular de un paradigma voseante. Aunque el uso de viste como marcador discursivo en el español rioplatense fue mencionado por Company Company (2004) entre otros marcadores discursivos deverbales, y se trata con mayor profundidad en el trabajo de Fløgstad (2012), no se ha realizado, hasta el momento, un análisis histórico que pudiera dar cuenta del desarrollo diacrónico de esta partícula.

El trabajo se estructura de la siguiente manera: a continuación, se definirá qué es un marcador discursivo y se enumerarán los distintos marcos 
teóricos que los autores han utilizado para analizar los procesos de creación de dichas partículas, así como los trabajos de investigación previos sobre este tema. Luego, en la sección 2, se indicarán las preguntas de investigación que guían este trabajo, las hipótesis planteadas considerando la teoría presentada y los trabajos existentes sobre la evolución diacrónica de formas que originaron marcadores discursivos, y se detallará la metodología empleada para realizar el análisis. En la sección 3, se presentarán los resultados obtenidos a partir del análisis estadístico y se relacionarán con las hipótesis del artículo. Finalmente, luego de comentar las limitaciones del estudio y posibles sugerencias para trabajos futuros, en la sección 4 se expondrán las conclusiones a las que se llegó tras el análisis de todo el material presentado a lo largo de la investigación

\subsection{Los marcadores discursivos}

Para lograr que los interlocutores interpreten los discursos en la forma en que pretende el hablante, este puede utilizar marcadores discursivos: partículas que organizan la información y conectan los enunciados del discurso entre sí y con el contexto en el que este discurso se produce (Martín Zorraquino y Portolés Lázaro 1999; Garcés Gómez 2008). Estas partículas derivan de otras clases de palabras, como verbos, adjetivos o adverbios. Sin embargo, cuando el hablante las utiliza como marcadores discursivos, adquieren determinadas características que las diferencian de las palabras de las que provienen cuando estas funcionan individualmente. A partir de los siguientes ejemplos, extraídos de corpus del español, es posible estudiar esta oposición. En (1), viste funciona como verbo principal de la oración en la que aparece: es el pretérito de la segunda persona del singular del verbo ver, una segunda persona que percibió algo con sus ojos:

(1) Nené, yo lo quiero ver al Panchito. ¿Cuándo lo viste vos? (Manuel Puig 1972, CORDE [Corpus Diacrónico del Español])

(2) Hay que dar oficios, viste, no hay que ser tan general. (Ernesto Sábato 1974, CORDE)

A diferencia de (1), donde viste claramente es parte de la estructura sintáctica de la oración, en (2), viste aparece como una aposición, sin ninguna función sintáctica dentro de la oración que contiene los enunciados que conecta. En este caso, viste funciona como marcador discursivo, más específicamente, como marcador conversacional de modalidad epistémica que se orienta hacia el interlocutor. Según la clasificación de Martín Zorraquino y Portolés Lázaro (1999), este tipo de marcadores se utiliza en enunciados asertivos para indicar la actitud del hablante hacia su discurso en relación con su oyente. En este caso, dado que viste proviene del pretérito de un verbo con significado evidencial (considerando que percibir algo con los ojos implica que quien lo hace tiene evidencia de la existencia de lo que percibe), la intención del hablante podría ser darle a entender a su interlocutor que su mensaje incluye contenido que él ya debería conocer porque tiene evidencia suficiente para aceptarlo. Por lo tanto, viste podría clasificarse como un marcador conversacional de evidencia, un tipo de marcadores que funciona como reforzador de la aserción del hablante (Martín Zorraquino y Portolés Lázaro 1999). Por ejemplo: 
(3) Como cuando se muere algún nato ¿viste? que se queda a apoliyar en el auto con el motor prendido y lo hace cagar el monóxido de carbono, creo. (Roberto Fontanarrosa 1995, CREA [Corpus de referencia del español actual])

(4) Los márgenes de los comercios son enormes. Siguen pensando que estamos con alta inflación, viste. Igual con los zapatos. Te piden 150 dólares y más. (Diario La Nación 1992, CREA)

El hablante utiliza viste en (3) porque asume que su interlocutor conoce la situación de la que está hablando, probablemente porque viven en la misma comunidad y el evento que relata es de público conocimiento. Algo similar sucede en (4), ejemplo en el que el hablante incluye viste por la presunción de que el interlocutor está al tanto de la situación económica del país y el impacto que esta tiene en los precios de los productos. En ambos casos, el hablante apela a la evidencia que, según él, tiene el interlocutor para reforzar su afirmación, darla como entendida y aceptada por el oyente, y poder continuar con su discurso.

El proceso diacrónico de creación de marcadores discursivos ha despertado mucho interés recientemente entre los académicos que analizan la lingüística histórica por los fenómenos que intervienen en el desarrollo de estas partículas. Varios autores han estudiado la creación de marcadores discursivos como resultado del mecanismo de subjetivización, al que consideran parte del proceso de gramaticalización (Company Company 2004; Fagard 2012; Lazo García 2016). Sin embargo, otros autores hacen hincapié en las diferencias entre el proceso de creación de marcadores discursivos y la gramaticalización (Brinton 2001; Waltereit 2002; Ocampo 2009).

A continuación, se definirán los distintos marcos teóricos en virtud de los cuales se ha analizado la creación de estas partículas y se explicarán los distintos análisis de autores que han estudiado el desarrollo diacrónico de marcadores discursivos que se observan principalmente en interacciones con un otro hacia quien se orienta el discurso. El objetivo principal de este trabajo es analizar el uso de viste en el español rioplatense y su posible evolución hasta convertirse en un marcador discursivo en la actualidad. Dado que la creación de marcadores discursivos surge de un proceso subjetivo de manipulación de las formas por parte de los hablantes, conocer el desarrollo diacrónico de estas formas permite entender cómo utilizan las personas la lengua a lo largo de distintas épocas y cómo se disemina dicho uso en una comunidad de hablantes.

\subsection{Gramaticalización}

Uno de los procesos mediante los cuales opera el cambio lingüístico es la gramaticalización (Company Company 2003, 2004; Crowley y Bowern 2010). La gramaticalización ocurre cuando "una forma o construcción asume una función gramatical, o una forma o construcción gramatical asume una función aún más gramatical” (Company Company 2003, 9). Las formas resultantes de este proceso adquieren un significado más abstracto, adquieren nuevas funciones gramaticales (que tal vez antes no tenían o se añaden a 
las funciones gramaticales existentes). Hopper y Traugott (1993) esbozaron una escala de gramaticalización (en inglés, cline of grammaticality) para ilustrar el proceso que atraviesan las formas gramaticalizadas:

palabra léxica $>$ palabra gramatical $>$ clítico $>$ afijo flexivo $(>\varnothing)$

Algunos autores sostienen que el fenómeno de gramaticalización es unidireccional, es decir, el cambio solo ocurre de izquierda a derecha de la escala anterior, desde formas léxicas a formas gramaticales (Lehmann 1995). Sin embargo, otros autores consideran que existen casos de gramaticalización con direccionalidad opuesta y que, si bien son menos los ejemplos que van en esta dirección, no se puede definir la unidireccionalidad de la gramaticalización como algo taxativo sino como una tendencia sólida (Hopper y Traugott 1993; Traugott 2001; Norde 2009; Meng 2019). En este grupo de cambios que parecerían tener resultados opuestos a los de la gramaticalización, se destacan aquellos cambios que van de formas gramaticales a formas léxicas con estatus menos gramatical; es decir, formas que pasan de la sintaxis al discurso, como la creación de marcadores discursivos (Company Company 2004).

Este proceso se ha estudiado dentro del marco teórico de la gramaticalización, pero muchos autores han notado que no se observan todas las características propias de este tipo de cambios (Fagard 2012; Brinton 2017): los marcadores discursivos son partículas opcionales que el hablante utiliza para guiar al oyente hacia una correcta interpretación de su discurso y para expresar su actitud hacia el contenido del mensaje. Esta definición de marcador discursivo es incompatible con los resultados de un proceso tradicional de gramaticalización. Por lo tanto, el estudio diacrónico de marcadores discursivos también se ha vinculado con otros procesos relacionados con la gramaticalización.

\subsubsection{Desgramaticalización}

Company Company (2004) utiliza este término para referirse a los cambios que muestran una dirección opuesta a la de la gramaticalización. Lehmann (1995) fue quien utilizó este término por primera vez para referirse a la unidireccionalidad de la gramaticalización y hacer hincapié en la no existencia de la desgramaticalización. Más adelante, la desgramaticalización se definió como un cambio compuesto en el que un morfema gramatical, en un contexto determinado, adquiere autonomía o incrementa su contenido en uno o más niveles lingüísticos (semántico, morfológico, sintáctico o fonológico), con tres características clave (Norde 2010).

En primer lugar, los cambios clasificados como desgramaticalización deben mostrar un trayecto opuesto al de Hopper y Traugott (1993) que se indicó más arriba. Aquí surge una de las principales diferencias entre ambos procesos: mientras la gramaticalización puede llegar hasta el último paso de la escala (es decir, la eliminación del morfema), no se registran casos de desgramaticalización que hayan llegado al extremo de la escala y que hayan resultado en un morfema autónomo (Norde 2010). Además, Norde (2010) indica que es necesario que exista un contexto ambiguo que dé lugar al 
reanálisis de la forma en cuestión y que la forma nueva sea completamente nueva, que no coexista con la forma a partir de la cual se originó. El último requisito que la autora plantea es la existencia de un cambio interno en la forma desgramaticalizada, por lo que considera que los usos metalingüísticos no serían ejemplos de desgramaticalización (Norde 2009). Esta definición de desgramaticalización no parece ser compatible con la creación de marcadores discursivos, dado que estas partículas no experimentan un cambio interno y coexisten con las formas que les dieron origen.

\subsubsection{Pragmaticalización}

Este proceso ocurre cuando un elemento proposicional, como expresiones adverbiales, desarrolla un significado más abstracto y adquiere un significado pragmático para pasar a operar a nivel discursivo y dejar de operar a nivel oracional (Defour et al. 2012; Brinton 2017). La diferencia entre pragmaticalización y gramaticalización radicaría en el hecho de que, mientras que las formas gramaticalizadas se convierten en obligatorias, las formas pragmaticalizadas son opcionales y son externas a la estructura oracional.

Sin embargo, la pragmaticalización comparte con la gramaticalización el proceso de manipulación subjetiva de las formas por parte del hablante (Company Company 2004; Brinton 2017). Por eso, algunos autores consideran que la pragmaticalización es parte de la gramaticalización si es que esta se considera en un sentido más amplio, como un proceso que opera en distintos niveles de la lengua (Company Company 2004; Brinton y Traugott 2005; Castillo Lluch 2006).

\subsubsection{Subjetivización}

Castillo Lluch (2006) considera este proceso como un sinónimo de pragmaticalización. En este sentido, por subjetivización se entiende la incorporación de "significados valorativos, subjetivos o intersubjetivos" a formas léxicas o gramaticales que, en su origen, eran objetivas (Castillo Lluch 2006, 1745). Según Company Company (2004), los hablantes subjetivizan verbos, proceso que resulta en marcadores discursivos, mediante el enriquecimiento pragmático y la codificación de sus valoraciones en la sintaxis de la lengua, sintaxis que se va perdiendo a medida que la forma se subjetiviza. Si esta inferencia personal se expande, se puede llegar a construir un significado convencional en la lengua (Company Company 2004).

Si bien la forma que atraviesa subjetivización experimenta un debilitamiento del significado original, típico de la gramaticalización, el resultado es una forma con mayor autonomía que no es parte de la estructura sintáctica oracional, característica de la desgramaticalización (Company Company 2004). Algunos autores sostienen que el proceso de subjetivización se diferencia de la gramaticalización por los resultados, aunque las motivaciones iniciales sean las mismas, dado que las formas resultantes necesariamente atraviesan un debilitamiento semántico para luego adquirir un nuevo significado pragmático más subjetivo que permita que la forma se aleje de la gramática de la lengua (Brinton 2001; Company Company 2004). 
Considerando la discusión teórica de los últimos años en relación con el proceso de creación de marcadores discursivos, cobra fuerza la idea de pensar a la gramaticalización como un proceso más complejo que simplemente la creación de partículas con funciones sintácticas; un proceso que opera en distintos niveles de la lengua (la sintaxis y la pragmática), en el que se tenga en cuenta que los usuarios son quienes hacen la gramática y quienes cambian constantemente la lengua, y que la pragmática es parte de la gramática (Traugott y Dasher 2002; Company Company 2004). Dado que la lengua no es algo estático porque está evolucionando constantemente, aunque los resultados de los procesos de pragmaticalización (o subjetivización) no se ajusten a la definición tradicional de gramática en un sentido estrecho, sería lógico considerarlos como mecanismos del proceso de gramaticalización, que debería entenderse en un sentido más amplio que el tradicional, como sostienen varios autores (Company Company 2004; Castillo Lluch 2006; Lazo García 2016).

\subsection{Estudios previos}

A partir de la gran flexibilidad de los verbos del español para ser recategorizados (Company Company 2004), varios autores han estudiado el proceso diacrónico de creación de marcadores discursivos deverbales en las últimas décadas. Entre estos estudios, se destacan aquellos que se ocupan de marcadores discursivos orientados al oyente. Azofra Sierra y Enghels (2017), por ejemplo, analizaron el desarrollo de sabes como marcador discursivo a partir de un verbo de cognición, saber, en el español peninsular con datos de entre finales del siglo XIX y principios del siglo XXI. En su artículo, las autoras consideran que el uso de sabes resulta de un proceso de gramaticalización entendido en sentido amplio, siguiendo la definición de Company Company (2004), quien señala que la gramaticalización es "un macrocambio dinámico, un cambio de cambios, que engloba distintas subclases y procesos [...] la rutinización o cristalización del uso, sea cual sea la direccionalidad del cambio" (citado en Azofra Sierra y Enghels 2017, 5). Siguiendo este criterio, Azofra Sierra y Enghels concluyen que sabes experimentó gramaticalización, dado que aumentó su uso, se redujo su complejidad sintáctica, se excluyó de la estructura oracional y atravesó un proceso de blanqueamiento semántico, clave para la expansión de las funciones pragmáticas de la forma.

En los trabajos sobre marcadores discursivos del español rioplatense se han abordado los marcadores derivados de verbos de percepción, particularmente de verbos de visión, como mirar o ver. Estos verbos, según señala Sweetser (1990), son propensos a desarrollar significados más abstractos relacionados con la actividad mental, cambio que ha ocurrido en varios idiomas y que, en algunas lenguas, ha resultado en marcadores discursivos (Waltereit 2002; Van Olmen 2012). Esta posibilidad de expansión semántica es lo que permite que los hablantes manipulen las formas para recargarlas de subjetividad. Entre estos trabajos sobre el español rioplatense se encuentra el de Ocampo (2009), en el que se analiza la evolución de mirá de verbo a marcador discursivo. El autor toma la base teórica de Martín Zorraquino y Portolés Lázaro (1999), quienes categorizan este marcador dentro de los marcadores conversacionales con función de enfocador de alteridad, 
partículas que apuntan al oyente. Si bien el marcador mirá comparte muchas propiedades con otros marcadores discursivos, en tanto no admite negación ni complementos, a diferencia de la mayoría de los marcadores discursivos, que son invariables, mirá sí admite variación según el grado de formalidad del hablante al que se esté apelando (Martín Zorraquino y Portolés Lázaro 1999, citado en Ocampo 2009). Además, Ocampo (2009) encontró que el marcador también se utiliza en la segunda persona del plural, miren.

Para explicar el marco teórico de su análisis, Ocampo (2009) refiere a su trabajo sobre la evolución diacrónica de adjetivo a marcador discursivo de claro, también con un corpus rioplatense (Ocampo 2006). En este trabajo, el autor indica que se adhiere a la postura presentada por Hopper y Traugott (1993, citado en Ocampo 2006) y sostiene que la gramaticalización es parte de un proceso más amplio de cambio semántico. En este proceso existen tres vértices: gramática, discurso y léxico, y las formas se mueven de uno a otro en un proceso fluido que no tiene una dirección obligatoria (Ocampo 2009). El autor llama discursivización al cambio mediante el cual elementos del léxico o la gramática pasan al ámbito del discurso, proceso que sigue la forma mirá. Ocampo (2009) indica que el significado original de mirar, dirigir la vista hacia un objeto, experimentó un debilitamiento semántico hasta adquirir un significado más abstracto: prestar atención. Es a partir de este significado que mirá adquiere usos discursivos (Ocampo 2009).

Otro trabajo sobre marcadores discursivos procedentes de verbos de percepción visual es el de Fløgstad (2012) sobre el uso de viste como marcador discursivo en el español del Río de la Plata. Con los resultados de un estudio previo sobre la expansión del pretérito simple en el español del Río de la Plata (Fløgstad 2014), la autora encontró un aumento en la frecuencia de uso de viste y reconoció que los hablantes rioplatenses utilizaban viste como marcador discursivo (Fløgstad 2012). Fløgstad indica que la forma viste atravesó el frecuente proceso de cambio semántico de visión física a conocimiento o visión mental que menciona Sweetser (1990, citado en Fløgstad 2012), como se indicó anteriormente. A continuación, se presentan ejemplos tomados de corpus electrónicos para ilustrar los tres usos de viste que menciona la autora en el análisis de sus datos:

(5) Le grité: “¿¡Por televisión lo viste!? ¡¿Por televisión!? (Diego Maradona 2000, CREA)

(6) Che, viste cómo me están pegando los diarios..., habría comentado el ministro [...]

(Diario Clarín 1997, CREA)

(7) Es mi manera de ser, ¿viste? Yo me subo a un subte vestido así y se produce un choque impresionante [...] (Carlos Polimeni 1991, CREA)

En (5), es posible ver que viste se usa con su significado original, dado que se hace referencia a la visión física, en este caso, de algo que apareció en la televisión. Sin embargo, en (6), el uso de viste no hace referencia a la percepción visual del hablante, sino al conocimiento de una situación. En este caso, viste se utiliza con un significado epistémico y podría ser reemplazado por verbos de cognición, como saber. Por último, en (7), el hablante 
utiliza viste como refuerzo de su aserción apelando al conocimiento que comparte con el oyente, quien evidentemente conoce la personalidad del hablante. Después de usar el marcador, probablemente para que no haya posibilidad de que se refute su declaración, el hablante hace explícito lo que supone que el oyente ya debería conocer.

Siguiendo el marco teórico que plantea Company Company (2011, citado en Fløgstad 2012), en su trabajo sobre viste, Fløgstad (2012) coincide con esta autora en que el proceso de creación de marcadores discursivos constituye un ejemplo que cuestiona la rígida definición tradicional de gramaticalización. Aunque existen características que este cambio comparte con la gramaticalización, como el debilitamiento semántico de la forma y la ampliación de su significado a uno más general, también se menciona que la evolución de verbo a marcador discursivo demuestra características que se oponen a la gramaticalización, como la autonomía sintáctica y la opcionalidad de la forma (Fløgstad 2012).

Si bien el trabajo de Fløgstad (2012) analizó la existencia del uso de viste como marcador discursivo en la comunidad de habla rioplatense, no existe, a la fecha, un estudio diacrónico sobre la evolución de la forma verbal viste hasta su uso como marcador discursivo. Dado que, como se indicó anteriormente, los verbos de percepción se consideran una fuente importante de marcadores discursivos en el español, particularmente el verbo ver (Company Company 2004), sería interesante complementar los hallazgos de Fløgstad (2012) para conocer el desarrollo diacrónico de este nuevo marcador del español rioplatense.

\section{Metodología}

\subsection{Objetivo, preguntas de investigación e hipótesis}

El objetivo de este trabajo es realizar un análisis diacrónico del uso de viste en el español rioplatense para analizar si experimentó un debilitamiento semántico que alejó la palabra de su significado etimológico evidencial hacia un significado epistémico que permitió su uso como marcador discursivo. Se busca responder las siguientes preguntas de investigación:

1) ¿Se observa, en los datos, un aumento en el uso de viste como verbo principal con significado epistémico a lo largo del siglo XX?

2) ¿Se observa, en los datos, un aumento en el uso de viste como marcador discursivo a lo largo del siglo XX?

Siguiendo la teoría analizada anteriormente y de acuerdo con las preguntas de investigación que guían este trabajo, se plantean las siguientes hipótesis:

\section{A) Análisis diacrónico del uso de viste como verbo principal}

En relación con la primera pregunta, como ocurre con todo cambio lingüístico, la frecuencia de uso de la forma innovadora aumenta con el paso del 
tiempo, períodos que, según el cambio, pueden ser tan cortos como veinte años (Camus Bergareche 2006) o extenderse por siglos (Hernández Díaz 2006). Dado que el significado epistémico de viste es innovador para este verbo, se supone que:

$$
\begin{aligned}
& \mathrm{H}_{\circ} \text { : En el transcurso del siglo XX, no se observa un aumento en el uso de viste como verbo } \\
& \text { principal con significado epistémico. } \\
& \mathrm{H}_{1} \text { : En el transcurso del siglo XX, se observa un aumento en el uso de viste como verbo } \\
& \text { principal con significado epistémico. }
\end{aligned}
$$

\section{B) Análisis diacrónico del uso de viste como marcador discursivo}

En cuanto a la segunda pregunta de investigación, como se indicó previamente, el proceso que atraviesa una forma gramatical para convertirse en un marcador discursivo se extiende a lo largo de un período de tiempo (Company Company 2004). Una vez que surge la nueva forma, tal como se explicó en el apartado anterior, con el transcurso del tiempo puede (o no) llegar a consolidarse. Por lo tanto, se espera que:

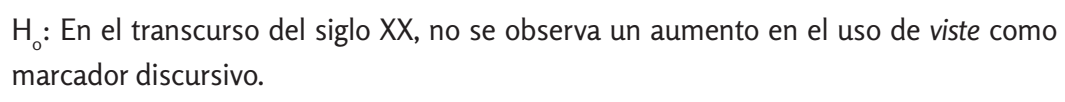

$H_{1}$ : En el transcurso del siglo XX, se observa un aumento en el uso de viste como marcador discursivo.

\subsection{Procedimiento}

Para responder a las preguntas de investigación y poder evaluar las hipótesis planteadas, en el análisis se utilizaron los datos pertenecientes a tres corpus electrónicos de la Real Academia Española: el Corpus Diacrónico del Español (CORDE), que comprende datos históricos hasta el año 1975; el Corpus del Español Actual (CREA), que incluye ejemplos que datan de entre 1975 y 2000; y el Corpus del Español del Siglo XXI (CORPES XXI), que está conformado por ejemplos obtenidos entre el año 2000 y 2016. Se realizó la búsqueda de la palabra viste en los sitios web de los tres corpus dentro de la categoría de español rioplatense. Dado que las versiones anotadas de CREA y CORPES incluyen los ejemplos de Argentina, Paraguay y Uruguay en su corpus del español rioplatense, se buscaron ejemplos en estos tres países en CORDE, ya que este corpus no divide los ejemplos por regiones geográficas y lingüísticas, sino por países individuales. Se descargaron todas las oraciones que incluían la palabra viste, y se descartaron todos aquellos casos en los que viste funciona como presente de la tercera persona singular del verbo vestir. Se obtuvo un total de 353 instancias de viste, en una muestra de 14.783 palabras. Los ejemplos se ordenaron cronológicamente y se clasificaron según su uso como verbo principal o como marcador discursivo. Una vez realizada la clasificación, se realizó el análisis de los datos en el programa de análisis estadístico Minitab. 
Para realizar el análisis estadístico se excluyeron los datos correspondientes a períodos anteriores al siglo XX: se encontraron solo cinco instancias de uso de viste (como pretérito de la segunda persona singular del verbo ver) en el siglo XIX y no se encontraron ejemplos correspondientes a épocas anteriores, por lo que la muestra es demasiado pequeña como para poder generalizar resultados para el siglo entero. Dado que los datos del siglo XXI obtenidos de CORPES XXI corresponden a entrevistas radiales o por televisión, a diferencia de los datos de CORDE y CREA que corresponden a ejemplos tomados de textos escritos, los datos de CORPES XXI se analizaron por separado. Para el análisis diacrónico se considera que, en muchos textos escritos, como textos literarios y cartas, la lengua se asemeja al discurso oral (Mosegaard Hansen y Rossari 2005). Sin embargo, teniendo en cuenta que el uso de marcadores discursivos es diferente en conversaciones orales espontáneas en comparación con los textos escritos porque la conversación suele favorecer la presencia de determinados marcadores (Martín Zorraquino y Portolés Lázaro 1999; Lindström y Wide 2005; Castillo Lluch 2006; Brinton 2017), se consideró apropiado separar los datos orales y escritos para que un posible uso más frecuente de marcadores discursivos en la oralidad no distorsionara los resultados de la investigación.

Los datos analizados extraídos de los dos corpus restantes corresponden al siglo XX. Para analizar la variación semántica en el uso de viste a lo largo del siglo XX, se dividió el siglo en cuatro partes. La división en cuatro etapas permite observar cómo se comportaba viste en distintos momentos del siglo XX para corroborar si su expansión semántica se dio de forma progresiva. A fin de evaluar el comportamiento diacrónico de viste según su función como marcador discursivo o verbo principal, para el análisis estadístico, se tomaron las dos mitades del siglo XX como unidades de análisis. Considerando que los usos de viste como marcador discursivo se han documentado como un fenómeno reciente y, en efecto, no se encontraron usos de viste como marcador discursivo en la primera mitad del siglo XX, estudiar las dos mitades del siglo XX permitirá corroborar si el cambio se dio efectivamente en la segunda mitad y si dicho cambio fue estadísticamente significativo.

\section{Resultados y discusión}

Para analizar los datos se utilizó el software Minitab, en el cual se realizó un análisis estadístico de los ejemplos utilizando la prueba chi-cuadrado, que permite analizar los datos de frecuencia de una muestra para evaluar la relación entre variables categóricas. También se analizó la correlación entre las

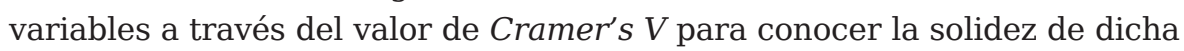
correlación (Gravetter y Wallnau 2013). Además, se calculó la prueba exacta de Fisher, que se utiliza en tablas de contingencia de 2 × 2 cuando la muestra es pequeña. Esta prueba se utilizó para garantizar la significancia estadística de los resultados, dado que la prueba chi-cuadrado no arroja resultados extremadamente confiables cuando se analizan muestras que no son tan grandes (The Pennsylvania State University 2018).Para todas las pruebas 
estadísticas se consideró un valor alpha de .05. A continuación, se muestran los resultados obtenidos en cuanto a la evolución del uso de viste como verbo principal con su significado original y con el significado innovador, y al uso diacrónico de viste como verbo principal y como marcador discursivo.

\subsection{Debilitamiento semántico de viste}

Se analizaron los distintos períodos en los que se dividió el corpus para evaluar si viste experimentó una expansión semántica hacia un significado más epistémico a lo largo del siglo XX, que sería el primer paso para permitir la manipulación pragmática de viste por parte de los hablantes como marcador discursivo. Como se observa en la tabla 1, el uso de viste con significado epistémico aumentó durante el período analizado, y en el último cuarto del siglo XX se puede ver cómo ambos usos son prácticamente equivalentes. Según la prueba chi-cuadrado de independencia para evaluar la relación entre los dos significados de viste, tal relación fue estadísticamente significativa, como se observa en la tabla 2.

\begin{tabular}{lcc}
\hline Período & Significado literal & Significado epistémico \\
\hline $1900-1925$ & $100 \%$ & $0 \%$ \\
\hline $1925-1950$ & $76 \%$ & $24 \%$ \\
\hline $1950-1975$ & $72 \%$ & $28 \%$ \\
\hline $1975-2000$ & $57 \%$ & $43 \%$ \\
\hline
\end{tabular}

Tabla 1. Usos de viste.

\begin{tabular}{lccc}
\hline Estadística & Valor & df & Sig. (2-tailed) \\
\hline Chi-cuadrado & 8.349 & 3 & 0.039 \\
\hline Cramer's V & 0.034 & & \\
\hline N.o de casos válidos & 242 & & \\
\hline
\end{tabular}

Tabla 2. Test estadístico.

A partir de las tablas anteriores, es posible confirmar la hipótesis H1B, dado que la forma viste experimentó un debilitamiento semántico que expandió su significado original evidencial hacia un significado epistémico y este uso innovador de viste, como verbo principal con significado epistémico, aumentó a lo largo del siglo XX de forma significativa.

\subsection{Evolución diacrónica de los usos de viste}

Se analizó la evolución cronológica de los usos de viste según su función como verbo principal y como marcador a lo largo de todo el siglo XX. Según se observa en la tabla 3 , si bien el uso de viste como verbo principal continúa siendo el más frecuente, se puede ver el surgimiento del uso de viste como marcador discursivo en la segunda mitad del siglo XX, que aumenta de forma considerable en relativamente poco tiempo. Sin ir más lejos, el primer uso de viste como marcador discursivo en el corpus estudiado data del año 1972, es decir, casi al final del tercer cuarto del siglo, lo que 
permitiría suponer que este uso innovador se expandió rápidamente entre los hablantes de español rioplatense en el último cuarto del siglo XX.

\begin{tabular}{lcc}
\hline Período & $\begin{array}{c}\text { Viste como } \\
\text { verbo principal }\end{array}$ & $\begin{array}{c}\text { Viste como marcador } \\
\text { discursivo }\end{array}$ \\
\hline $1900-1950$ & $100 \%$ & $0 \%$ \\
\hline $1950-2000$ & $81 \%$ & $19 \%$ \\
\hline
\end{tabular}

Tabla 3. Usos verbales y discursivos de viste.

Para evaluar la relación entre los distintos usos de viste, como verbo principal o marcador discursivo, en los períodos en los que se dividió el corpus se realizó una prueba de chi-cuadrado de independencia. La relación entre las variables fue significativa, como se observa en la tabla 4 . Además, dado que la tabla de contingencia para esta prueba es de 2 x 2 (en este caso, dos usos de viste en dos períodos diferentes), fue posible realizar una prueba exacta de Fisher para confirmar el carácter significativo del resultado.

\begin{tabular}{lccc}
\hline Estadística & Valor & df & Sig. (2-tailed) \\
\hline Chi-cuadrado & 5.110 & 1 & 0.024 \\
\hline Prueba exacta de Fisher & & & 0.018 \\
\hline N. o de casos válidos & 294 & & \\
\hline
\end{tabular}

Tabla 4. Test estadístico.

Considerando los resultados obtenidos a partir del análisis precedente, se puede confirmar la hipótesis $\mathrm{H1C}$, ya que se puede ver claramente un rápido aumento en el uso de viste como marcador discursivo en la segunda mitad del siglo XX. El uso de viste como marcador discursivo surge una vez consolidado el uso de viste como verbo principal con significado epistémico, por lo que se podría asumir que el debilitamiento semántico de viste dio lugar a que la forma se recategorizara como marcador discursivo.

Las 54 instancias de viste que se pudo obtener de CORPES XXI no se pudieron dividir en diversos períodos, ya que corresponden a datos que datan del período 2002 - 2016. A fin de analizar los datos obtenidos de CORPES XXI, se realizó una prueba chi-cuadrado de bondad de ajuste. Esta prueba se realiza para conocer la forma de la distribución de una población determinada y saber qué tan bien se ajustan los datos de la muestra a los valores esperados en la hipótesis nula (Gravetter y Wallnau 2013). Así, fue posible analizar la distribución del uso de viste (como marcador discursivo o como verbo principal) en los datos obtenidos correspondientes al período 2002-2016, que se muestran en la tabla 5 .

\begin{tabular}{lcc}
\hline Período & Viste como verbo principal & Viste como marcador discursivo \\
\hline $2002-2016$ & $26 \%$ & $74 \%$ \\
\hline
\end{tabular}

Tabla 5. Usos verbales y discursivos de viste. 
Según la prueba chi-cuadrado de bondad de ajuste, la diferencia entre ambos usos de viste en este período es estadísticamente significativa, como se indica en la tabla 6 .

\begin{tabular}{lccc}
\hline Estadística & Valor & df & Sig. (2-tailed) \\
\hline Chi-cuadrado & 12.5185 & 1 & 0.000 \\
\hline N.o de casos válidos & 54 & & \\
\hline
\end{tabular}

Tabla 6. Test estadístico.

Estos resultados parecerían respaldar nuevamente la hipótesis H1C, dado que se observa un incremento, incluso una primacía, del uso de viste como marcador discursivo en este período. Sin embargo, como se indicó anteriormente, es necesario tener en cuenta que el uso de marcadores como viste es más frecuente en la oralidad, por lo que un uso más frecuente de viste como marcador discursivo puede deberse tanto a un aumento en el uso por la propia evolución diacrónica de la lengua como al hecho de que los datos corresponden a discursos orales.

\subsection{Limitaciones y estudios futuros}

Como en todos los trabajos en los que se realiza un estudio diacrónico de la lengua, en este trabajo también se encontraron limitaciones a la hora de obtener los datos necesarios en los que se documenta el fenómeno que se analiza (Labov 1994; Eska y Ringe 2013). Además, considerando que el uso de marcadores discursivos es más frecuente en la oralidad, y teniendo en cuenta que una de las limitaciones inherentes a los estudios de lingüística histórica es la falta de registros auditivos (Labov 1994), los resultados obtenidos deberían reforzarse con nuevos estudios en el futuro que permitieran analizar este fenómeno desde un punto de vista sociolingüístico para saber si algún factor externo influye en este proceso. Por ejemplo, se podrían considerar otras bases de datos, como el corpus PRESEEA, que considera "la diversidad sociolingüística de las comunidades de habla hispanohablantes" (PRESEEA 2014).

Dado que el uso de viste como marcador discursivo parece ser una innovación del siglo XX, especialmente durante la segunda mitad, sería posible analizar registros de películas, entrevistas o programas radiales de la época; en Argentina, donde se encuentra la mayoría de la población de hablantes de español rioplatense, la primera transmisión televisiva se realizó en 1951 (Otero 2016) y la primera película sonora data del año 1933 (Ministerio de Cultura 2020). De esta forma, con datos orales más antiguos, se podría realizar un análisis que le otorgara más solidez a los resultados de este estudio.

Otro aspecto que merece estudio es el hecho de que viste también se puede usar como marcador discursivo en la segunda persona singular formal, vio, o en la segunda persona plural, vieron (Fløgstad 2012). Esta variabilidad que sugiere Fløgstad, según lo que arrojan sus datos, pondría en duda la definición de viste como marcador discursivo, ya que, por su definición, los marcadores discursivos son formas invariables (Garcés Gómez 2008). Por ejemplo, los marcadores vaya, venga, anda, vamos, etc. están 
completamente gramaticalizados y no admiten flexión de persona (Castillo Lluch 2006). Sin embargo, algunos autores consideran que existen marcadores discursivos que sí admiten variación, como es el caso de los marcadores derivados del verbo mirar: mira, mirá, mire, miren (Martín Zorraquino y Portolés Lázaro 1999; Company Company 2004; Ocampo 2009).

Por lo tanto, sería interesante incorporar al análisis diacrónico el uso de vio y de vieron para saber si estas partículas se utilizan con la misma función y en la misma frecuencia que viste. Si se observara una diferencia en favor del uso de viste, se podría argumentar que las tres formas compitieron en algún momento (y tal vez lo hacen también en la actualidad), pero los hablantes habrían elegido como marcador discursivo universal la forma viste, que estaría camino a su completa gramaticalización. Este ejemplo parecería ilustrar esta situación:

(8) ¿Viste? Perdón, maestro. ¿Vio? ¡Qué pueblo! Y son rascas no más, dejan de comer para venir a oírlo. (Marta Lynch 1977, CREA)

En (8), el hablante utiliza viste, pero luego se da cuenta de que su interlocutor merece un trato más formal, por lo que corrige su discurso y utiliza vio. Este caso serviría para ilustrar el extenso uso de viste como marcador, que ya parecería haber pasado al sistema lingüístico de los hablantes rioplatenses como única forma de ver que se puede usar en esta función, sin importar la formalidad que requiera la situación lingüística en la que se encuentre el hablante. Este fenómeno podría estar relacionado con la generalización del tratamiento informal que ocurrió en Argentina (Kempas 2019), particularmente en la zona del Río de la Plata, donde "es común que los jóvenes voseen a los adultos no solo cuando existen relaciones familiares sino incluso cuando no hay previo conocimiento" (Carricaburo 1997, 24) ${ }^{1}$.

\section{Conclusión}

En este trabajo, se analizaron tres corpus electrónicos de la Real Academia Española con datos del período comprendido entre 1906 y 2016 a fin de estudiar la evolución diacrónica de viste como marcador discursivo en el español rioplatense. Para conocer el desarrollo de esta partícula, se analizaron los usos de viste como verbo principal y como marcador discursivo a lo largo de todo el período estudiado. Además, se prestó especial atención al significado de viste en su uso como verbo principal: se demostró que viste experimentó un debilitamiento semántico de su significado etimológico evidencial en favor de un significado epistémico.

Si bien ambos usos conviven en la actualidad en la variedad de español analizada, a lo largo del siglo XX, según se notó en los datos extraídos del corpus, se observó un aumento del uso de viste con significado epistémico. El debilitamiento semántico, paso obligatorio de todo proceso de

1 Sería interesante realizar un análisis histórico de dicha generalización para conocer si algún evento determinante para la sociedad, como la dictadura militar o la vuelta a la democracia en 1983, tuvo un papel primordial en la expansión del tratamiento informal por sobre el formal. 
gramaticalización, permitió que los hablantes pudieran manipular la forma pragmáticamente en su discurso y cargarla de subjetividad. Esto habilitó el uso de viste como marcador discursivo para expresar su suposición de que el interlocutor debe estar familiarizado con el contenido del mensaje y, así, reforzar su aserción.

A partir de los primeros usos documentados de viste como marcador discursivo en el corpus utilizado en este trabajo, se observó un aumento en su frecuencia de uso, especialmente en las últimas décadas del siglo XX. Aunque los marcadores discursivos no pertenecen a la estructura sintáctica oracional y son formas más autónomas que las que resultan de un proceso de gramaticalización, el proceso de desarrollo de estas partículas sí comparte algunas características básicas con los procesos de creación de gramática, como el proceso de debilitamiento semántico que parece haber sido clave en el desarrollo de viste como marcador discursivo del español del Río de la Plata.

La forma viste había sido descrita y estudiada como marcador discursivo de la variedad de español rioplatense (Company Company 2004; Fløgstad 2012), pero el proceso histórico mediante el cual se originó no se había analizado anteriormente. Este trabajo intenta continuar la conversación completando ese vacío y permite comprender de mejor manera los procesos mediante los cuales se forman estas partículas. De igual modo, analizar la formación histórica de un marcador discursivo como viste contribuye al estudio de la gramaticalización y otros procesos relacionados con esta, como la subjetivización, la pragmaticalización y la desgramaticalización, lo que permite ahondar en las hipótesis sobre el cambio lingüístico. 


\section{Q Bibliografía}

" Azofra Sierra, Elena y Renata Enghels. 2017. "El proceso de gramaticalización del marcador epistémico deverbal sabes”. Iberoromania 85: 105-129.

"Brinton, Laurel J. 2017. The evolution of pragmatic markers in English: Pathways of change. New York: Cambridge University Press.

» Brinton, Laurel J. 2001. "From matrix clause to pragmatic marker: The history of lookforms". Journal of Historical Pragmatics 2, 2: 177-199.

» Brinton, Laurel J. y Elizabeth C. Traugott. 2005. Lexicalization and Language Change. New York: Cambridge University Press.

" Camus Bergareche, Bruno. 2006. “La expresión de la negación. Sintaxis histórica de la lengua española”. En Sintaxis histórica de la lengua española, editado por Concepción Company Company, 1165-1252. México: Fondo de Cultura Económica.

»Carricaburo, Norma. 1997. Las fórmulas de tratamiento en el español actual. Madrid: Arco Libros.

» Castillo Lluch, Mónica. 2006. "La formación de los marcadores discursivos vaya, venga, anda y vamos". En Actas del VII Congreso Internacional de Historia de la Lengua Española, editado por Concepción Company Company y José G. Moreno de Alba, 1739-1752. Madrid: Arco Libros.

»Company Company, Concepción. 2011. "Principios teóricos vs. datos de corpus: ¿Diálogo o enfrentamiento? La formación de marcadores del discurso en -mente”. En Marcadores del discurso: Perspectivas y contrastes, editado por María Marta García Negroni, 13-34. Buenos Aires: Santiago Arcos.

»Company Company, Concepción. 2004. “¿Gramaticalización o desgramaticalización? Reanálisis y subjetivización de verbos como marcadores discursivos en la historia del español". Revista de Filología Española 84, 1: 29-66.

" Company Company, Concepción. 2003. "La gramaticalización en la historia del español". Medievalia 35: 3-61.

"Crowley, Terry y Claire Bowern. 2010. An introduction to historical linguistics. New York: Oxford University Press.

»Defour, Tine, Ulrique D'Hondt, Anne-Marie Simon-Vandenbergen y Dominique Willems. 2012. "Degrees of pragmaticalization: The divergent histories of 'actually' and actuellement". En Pragmatic Markers and Pragmaticalization: Lessons from False Friends, editado por Peter Lauwers, Gudrun Vanderbauwhede y Stijn Verleyen, 37-64. Amsterdam/Philadelphia: John Benjamins Publishing Company.

» Eska, Joseph F. y Donald Ringe. 2013. Historical linguistics. Cambridge: Cambridge University Press.

» Fagard, Benjamin. 2012. "É vida, olha...: Imperatives as discourse markers and grammaticalization paths". En Pragmatic Markers and Pragmaticalization: Lessons from False Friends, editado por Peter Lauwers, Stijn Verleyen y Gudrun Vanderbauwhede, 117-140. Amsterdam/Philadelphia: John Benjamins Publishing Company.

" Fløgstad, Guro. 2014. "The expansion of the preterit in Rioplatense Spanish: Contact induced?”. En The Sociolinguistics of Grammar, editado por Tor A. Afarli y Brit Mæhlum, 117-136. Amsterdam/Philadelphia: John Benjamins Publishing Company. 
» Fløgstad, Guro. 2012. “Viste: ¿Un caso de gramaticalización?”. En Actas del II Coloquio Internacional Marcadores del discurso en lenguas románicas: un enfoque contrastivo, editado por María Marta García Negroni, 82-88. Buenos Aires: Editorial de la Facultad de Filosofía y Letras de la Universidad de Buenos Aires.

» Fontanella de Weinberg, María B. 1999. “Sistemas pronominales de tratamiento usados en el mundo hispánico”. En Gramática descriptiva de la lengua española, editado por Ignacio Bosque y Violeta Demonte, 1399-1425. Madrid: Espasa Calpe.

» Fraser, Bruce. 2009. “An account of discourse markers”. International Review of Pragmatics 1,2: 293-320.

» Garcés Gómez, María P. 2008. La organización del discurso: marcadores de ordenación y de reformulación. Madrid: Iberoamericana/Vervuert.

» García Negroni, María Marta y Silvia Ramírez Gelbes. 2010. “Acerca del voseo en los manuales escolares argentinos (1970-2004)”. En Formas y fórmulas de tratamiento en el mundo hispánico, editado por Martin Hummel, Bettina Kluge y María Eugenia Vázquez Laslop, 1013-1032. México: El Colegio de México

» Gravetter, Frederick J. y Larry B. Wallnau. 2013. Statistics for behavioral sciences. 9ª . ed. Belmont: Wadsworth.

» Hernández Díaz, Axel. 2006. “Posesión y existencia. La competencia de haber y tener y haber existencial”. En Sintaxis histórica de la lengua española, editado por Concepción Company Company: 1055-1164. México: Fondo de Cultura Económica.

» Hopper, Paul J. y Elizabeth C. Traugott. 1993. Grammaticalization. Cambridge: Cambridge University Press.

» Kempas, Ilpo. 2019. “La elección de las formas de tratamiento en las páginas de tiendas online: Un recorrido por el mundo hispano”. Bergen Language and Linguistic Studies 10, 1: 1-14. https://doi.org/10.15845/bells.v10i1.1339

» Lazo García, Verónica J. 2016. “El cambio lingüístico por subjetivización: el caso de ‘digamos”". Escritura y Pensamiento 19, 39: 213-234.

» Labov, William. 1994. Principles of Linguistic Change. Internal factors. Oxford: Blackwell Publishing.

» Lehmann, Christian. 1995. Thoughts on grammaticalization. Berlin: Language Science Press.

" Lindström, Jam y Camilla Wide. 2005. "Tracing the origins of a set of discourse particles: Swedish particles of the type you know". Journal of Historical Pragmatics 6, 2: 211-236.

» Martín Zorraquino, María. A. y José Portolés Lázaro. 1999. “Los marcadores del discurso”. En Gramática descriptiva de la lengua española Vol. 3, editado por Ignacio Bosque y Violeta Demonte: 4051-4213. Madrid: Espasa Calpe.

» Meng, Qing Liang. 2019. “A corpus-based investigation of diachronic evolution of like and its variants through grammaticalization". Advances in Language and Literary Studies 10, 3: 81-88.

» Ministerio de Cultura. 2020. “Tango", la primera película sonora argentina, a 87 años de su estreno. Ministerio de Cultura Argentina. https://www.cultura.gob.ar/tango-la-primera-pelicula-sonora-de-la-argentina-a-85-anos-de-su-estreno_5721/

» Mondaca Becerra, Lissette, Andrea Méndez Carrasco y Marcela Rivadeneira Valenzuela. 2015. “'No es muletilla, es marcador, ¿cachái?’: Análisis de la función pragmática del marcador discursivo conversacional cachái en el español de Chile”. Literatura y lingüística 32: 233-258. 
" Mosegaard Hansen, Maj-Britt y Corinne Rossari. 2005. The evolution of pragmatic markers. Amsterdam/Philadelphia: John Benjamins Publishing Company.

» Norde, Muriel. 2010. "Degrammaticalization: three common controversias”. En Grammaticalization: Current views and issues, editado por

" Katerina Stathi, Elke Gehweiler y Ekkehard König, 123-150. Amsterdam/Philadelphia: John Benjamins Publishing Company.

"Norde, Muriel. 2009. Degrammaticalization. Oxford: Oxford University Press.

"Ocampo, Francisco. 2009. "Mirá: From verb to discourse particle in Rioplatense Spanish". En Selected Proceedings of the 11th Hispanic Linguistics Symposium, editado por Joseph Collentine, Maryellen García, Francisco A. Marcos Marín y Barbara Armstrong Lafford, 254-267. Sommerville: Cascadilla Proceedings Project.

"Ocampo, Francisco. 2006. "Movement towards discourse is not grammaticalization: The evolution of claro from adjective to discourse particle in spoken Spanish". En Selected proceedings of the gth Hispanic Linguistics Symposium, editado por Nuria Sagarra y Almeida Jacqueline Toribio, 308-19. Sommerville: Cascadilla Proceedings Project.

»Otero, Nieves. 2016. "65 años de televisión en la Argentina: ¿cómo se gestó la primera transmisión?”. Infobae, 17 de octubre. https://www.infobae.com/teleshow/ infoshow/2016/10/17/65-anos-de-television-en-la-argentina-como-se-gesto-la-primeratransmision/

»Portolés Lázaro, José. 2015. “Los marcadores del discurso". En Enciclopedia Lingüística Hispánica, editado por Javier Gutiérrez-Rexach, 689-699. Londres/ Nueva York: Routledge.

» PRESEEA. 2014. Corpus del Proyecto para el estudio sociolingüístico del español de España y de América. Alcalá de Henares: Universidad de Alcalá. Fecha de consulta: 18 de julio 21. http://preseea.linguas.net

"Real Academia Española: Banco de datos (CORDE) [en línea]. Corpus diacrónico del español. http://corpus.rae.es/cordenet.html

》Real Academia Española: Banco de datos (CREA) [en línea]. Corpus de referencia del español actual. Versión anotada. https://webfrl.rae.es/CREA/view/inicioExterno.view;js essionid=7B9oBF20 $533 \mathrm{~F}_{1} \mathrm{BBBE}_{7} \mathrm{C}_{6 \mathrm{~A}} \mathrm{D}_{0155} \mathrm{E}_{4} \mathrm{FB}_{4}$

»Real Academia Española: Banco de datos (CORPES XXI) [en línea]. Corpus del español del siglo XXI.

" https://webfrl.rae.es/CORPES/view/inicioExterno.view;jsessionid=DED9D876BF1A3DE E20770B6C014F5425

"Sweetser, Eve. 1990. From etymology to pragmatics: Metaphorical and cultural aspects of semantic structure. Cambridge: Cambridge University Press.

"Tanghe, Sanne. 2016. "Position and polyfunctionality of discourse markers: The case of Spanish markers derived from motion verbs". Journal of Pragmatics 93: 16-31.

"The Pennsylvania State University. 2018. Two-way Tables - Exact Tests.

" https://online.stat.psu.edu/stat5o4/lesson/3/3.3

"Traugott, Elizabeth. C. 2001. “Legitimate counterexamples to unidirectionality”. Trabajo presentado en la Universidad de Freiburg, 17 de octubre.

» Traugott, Elizabeth C. y Richard B. Dasher. 2002. Regularity in semantic change. Cambridge: Cambridge University Press. 
» Van Olmen, Daniël. 2012. "The imperative of intentional visual perception as a pragmatic marker: A contrastive study of Dutch, English and Romance". En Pragmatic Markers and Pragmaticalization: Lessons from False Friends, editado por Peter Lauwers, Stijn Verleyen y Gudrun Vanderbauwhede, 95-115. Amsterdam/ Philadelphia: John Benjamins Publishing Company.

"Vincent, Diane. 2005. "The journey of non-standard discourse markers in Quebec French". Journal of Historical Pragmatics 6, 2: 188-210.

"Waltereit, Richard. 2002. "Imperatives, interruption in conversation, and the rise of discourse markers: a study of Italian guarda". Linguistics 40, 5: 987-1010. 